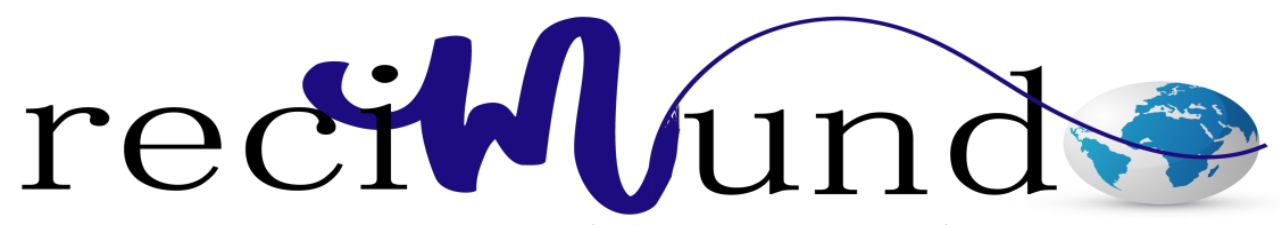

Revista Cientifica Mundo de la Investigación y el Conocimiento

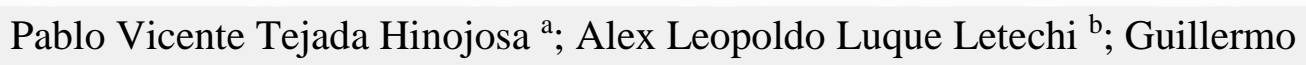
Roberto Abad Alvarado ${ }^{\mathrm{c}}$

La generación y administración de los recursos públicos en Ecuador, una perspectiva general

Revista Científica Mundo de la Investigación y el Conocimiento. Vol. 2 núm., 1, febrero, ISSN: 2588-073X, 2018, pp. 246-261

DOI: $10.26820 /$ recimundo/2.1.2018.246-261

Editorial Saberes del Conocimiento

Recibido: 01/12/2017

Aceptado: 15/02/2018
a. Universidad de Guayaquil; pablo.tejadah@ug.edu.ec
b. Universidad de Guayaquil; alex.luquel@ug.edu.ec
c. Universidad de Guayaquil; guillermo.abad@ug.edu.ec 


\section{La generación y administración de los recursos públicos en Ecuador, una \\ perspectiva general}

Vol. 2, núm. 1., (2018)

Pablo Vicente Tejada Hinojosa; Alex Leopoldo Luque Letechi; Guillermo Roberto Abad

Alvarado

\section{RESUMEN}

La recaudación de tributos, la concientización tributaria y la administración eficiente de los mismos es crucial para el buen desenvolvimiento de la gestión pública. Para ello todos los involucrados deben poner su empeño en la consecución de sus objetivos, en primer lugar el Estado debe velar por que los ciudadanos contribuyan con su deber de cancelar impuestos, en segundo lugar los ciudadanos deben velar por el buen uso por parte del Estado de dichos recursos y en tercer lugar, se deben generar los mecanismos para la optimización tanto del cobro como de los procesos de auditorías y control fiscal. La metodología empleada en la investigación es de tipo documental no experimental. Entre las conclusiones más relevantes es que según los datos obtenidos del sistema de rentas internas de Ecuador (SRI), si bien en base a las reformas generadas por el Estado Ecuatoriano para el impulso del crecimiento de recaudación de tributos, todavía no hay crecimientos sostenidos en el tiempo, a la par de que las estadísticas son publicadas en dólares y no en cantidad de contribuyentes que cumplen con su deber tributario. Como recomendaciones generales se destacan la creación de conciencia tributara en los ciudadanos, seguir implementando y estimulando el pago electrónico y capacitación continua y permanente de los administradores de recursos públicos.

Palabras clave: Recursos, Administración, dinero, Tributo, publico. 


\section{La generación y administración de los recursos públicos en Ecuador, una perspectiva general}

Vol. 2, núm. 1., (2018)

Pablo Vicente Tejada Hinojosa; Alex Leopoldo Luque Letechi; Guillermo Roberto Abad Alvarado

\section{ABSTRACT}

The collection of taxes, tax awareness and the efficient administration of taxes is crucial for the good development of public management. For this, all involved must put their efforts in achieving their objectives, first of all the State must ensure that citizens contribute their duty to pay taxes, secondly citizens must ensure the proper use by the State of these resources and thirdly, mechanisms must be generated for the optimization of both the collection and audit processes and fiscal control. The methodology used in the research is non-experimental documentary type. Among the most relevant conclusions is that according to the data obtained from the internal revenue system of Ecuador (SRI), although based on the reforms generated by the Ecuadorian State to boost the growth of tax collection, there is still no sustained growth in time, at the same time that the statistics are published in dollars and not in the number of taxpayers who fulfill their tax duty. As general recommendations we highlight the creation of tax awareness in the citizens, continue to implement and encourage electronic payment and continuous and permanent training of public resource managers.

Keywords: Resources, Administration, money, Tribute, public. 


\section{La generación y administración de los recursos públicos en Ecuador, una perspectiva general}

Vol. 2, núm. 1., (2018)

Pablo Vicente Tejada Hinojosa; Alex Leopoldo Luque Letechi; Guillermo Roberto Abad

\section{Introducción.}

Alvarado

El Estado Ecuatoriano para garantizar a sus habitantes la vida, la libertad, la justicia, la seguridad, la paz y el desarrollo integral de la sociedad, requieren generar recursos con el fin primordial de satisfacer las necesidades públicas; por lo que, la tributación es la forma de obtener dichos ingresos para el cumplimiento de sus fines sociales, asimismo, las leyes fiscales deben ser armónicas y unitarias, las que deben sujetarse tanto a la Constitución como a las leyes fiscales (Código Tributario, Ley Orgánica de Aduanas), para uniformar los procedimientos que son aplicables en forma general a cualquier tributo, para evitar la contradicción, repetición y falta de técnica legislativa en las leyes (Blacio, 2010). No es solo el tema de la recaudación o generación de dinero para satisfacer necesidades, debe haber un control de esos dineros públicos para que sean utilizados de la manera correcta.

En Ecuador, desde el mes de octubre del año 2010, está vigente el Código Orgánico de Ordenamiento Territorial y Descentralización (COOTAD), cuerpo legal que regula a los gobiernos autónomos descentralizados GADs, y en lo referente a los recursos financieros; establece como norma general y en concordancia con lo previsto en la Constitución, que "los gobiernos autónomos descentralizados generarán sus propios recursos financieros y, como parte del Estado, participarán de sus rentas, de conformidad con los principios de subsidiariedad, solidaridad y equidad interterritorial" (COOTAD, Art.163) (Oyervide, 2012). Es decir, se parte del proceso de auto gestión municipal, la capacidad que deben tener los gobiernos locales para generar sus propios ingresos en este caso por medio de los impuestos municipales y otros ingresos, y como administrarlos de la mejor manera para responder a las necesidades locales. 


\section{La generación y administración de los recursos públicos en Ecuador, una perspectiva general}

Vol. 2, núm. 1., (2018)

Pablo Vicente Tejada Hinojosa; Alex Leopoldo Luque Letechi; Guillermo Roberto Abad Alvarado

Una de las problemáticas más delicadas e importantes que plantea la descentralización es

el financiamiento de las actividades que deben desarrollar los entes descentralizados. Esto merece un especial énfasis porque hablar de descentralización sin asignación de recursos implica referirse no sólo a algo del todo incompleto y desnaturalizado sino, aún más, a un proceso al que le faltará practicidad, o bien que no logrará cumplida realización (Benalcázar, 2013). Porque la descentralización implica en gran medida la separación financiera con el Estado, aun cuando este de alguna manera continúe asignando recursos aguas abajo, en gran parte los gobiernos locales deben ser generadores de la mayor parte de sus propios recursos.

La palabra tributo proviene de la voz latina tributum que significa carga, gravamen, imposición; la misma, que era utilizada en la antigua Roma hasta el año 168 antes de Cristo, para asignar el impuesto que debía pagar cada ciudadano de acuerdo con el censo, y que era recaudado por tribus (Blacio, 2010).

Según la Real Academia Española de la Lengua, el término “administración” significa, acción y efecto de administrar y "administrar" proviene del verbo latino "administrare" que quiere decir servir, gobernar o ejercer algún cargo. Además el término "administrare" etimológicamente está constituido por las raíces latinas de "ad", "manus" y "tráhere" todo esto significa prestación de servicios, es decir, de traer a mano algo. Por lo que en su esencia es la actividad subordinada de quien sirve a favor de su beneficiario (Abad \& Paúl, 2012).

Para definir a la administración con el ente público es necesario mencionar al Dr. Hugo Settembrino, el cual nos aclara que "es una organización que el Estado utiliza para canalizar adecuadamente demandas sociales y satisfacerlas, a través de la transformación de recursos 


\section{La generación y administración de los recursos públicos en Ecuador, una perspectiva general}

Vol. 2, núm. 1., (2018)

Pablo Vicente Tejada Hinojosa; Alex Leopoldo Luque Letechi; Guillermo Roberto Abad

Alvarado públicos en acciones modificadoras de la realidad, mediante la producción de bienes, servicios y regulaciones"3 (Abad \& Paúl, 2012).

Según lo especificado en la Ley Orgánica de Régimen Tributario Interno (LORTI) ecuatoriano, los tributos "son ingresos públicos, creados por ley, en base de la capacidad contributiva del pueblo y encaminado a darle recursos al Estado para prestar servicios públicos que satisfagan las necesidades colectivas". Se dividen en:

- Tasa. - generada por la prestación de un servicio efectivo o potencial, brindado de manera directa por el Estado. Ejemplo: valor que se cancela por obtener la cédula de identidad.

- Impuestos. - contribuciones obligatorias establecidas por Ley, que deben pagar las personas naturales y las sociedades que se encuentran en las condiciones previstas por la misma. En resumen, los impuestos son el precio por vivir en una sociedad civilizada.

Por tanto, se considera como la principal obligatoriedad del Estado la construcción progresiva de un sistema tributario estable, justo y sostenible; así también a las empresas como entes económicos y a la sociedad quienes deben cumplir con su papel de contribuyentes, en función de su capacidad y carga impositiva (Fernanda, Letty, \& Marco, 2018). Es decir, hay una relación que debería ser directa entre las obligaciones que tiene el Estado en sus diferentes niveles de administrar los recursos financieros para dar respuestas a la sociedad en la producción de bienes y servicios y los ciudadanos tienen la obligación de aportar por medio de sus impuestos en la consecución de los proyectos y planes que tenga a bien realizar el Estado, es resumen Estado y ciudadano deben estar alineados en un objetivo común. 


\section{La generación y administración de los recursos públicos en Ecuador, una perspectiva general}

Vol. 2, núm. 1., (2018)

Pablo Vicente Tejada Hinojosa; Alex Leopoldo Luque Letechi; Guillermo Roberto Abad Alvarado

El manejo de las finanzas públicas se ha caracterizado por tener un bajo nivel de planificación, un alto contenido de gasto corriente y un endeble sistema de control. Generalmente, los recurrentes problemas de dificultad financiera se tienden a resolver mediante reformas tributarias, que en su esencia buscan controlar la evasión fiscal, recurriendo a novedosos impuestos o a modificar los existentes. A pesar de los graves impactos económicos y sociales del déficit fiscal, de la precaria gestión de las finanzas públicas, existe una alarmante inconsistencia colectiva sobre el tema. Indiferencia, desconocimiento y ligereza caracterizan al tratamiento de la realidad fiscal (Reyes, 2005). Una de las principales causas que generan la evasión fiscal es la desconfianza en el uso del dinero público que tiene el ciudadano (contribuyente), Ecuador no escapa a esto, el mal manejo, la corrupción y la burocracia están a la orden del día y esto desestimula el compromiso que tiene el ciudadano en su deber y derecho de pagar sus impuestos.

En la presente investigación se trata de hacer un abordaje general en lo que tiene que ver con el manejo de los dineros públicos y la generación de estos en Ecuador. Haciéndonos las siguientes preguntas: ¿para que se recauda?, ¿cuál es el deber del ciudadano?, ¿cómo debe ser el manejo de los recursos?.

\section{Materiales y Métodos}

La investigación aquí presentada está enmarcada dentro una investigación de tipo documental, no experimental, en vista de que los análisis aquí presentados están realizados en base a artículos de opinión, revistas, obtenidos de medios electrónicos y los cuales fueron utilizados como referencias bibliográficas, en ningún momento dentro de la investigación se ha 


\section{La generación y administración de los recursos públicos en Ecuador, una}

perspectiva general

Vol. 2, núm. 1., (2018)

Pablo Vicente Tejada Hinojosa; Alex Leopoldo Luque Letechi; Guillermo Roberto Abad

Alvarado

alterado alguna referencia, las opiniones aquí expuestas son de los autores referenciados, siendo solo opiniones del autor los análisis correspondientes.

\section{Resultados.}

Tabla Nº1: Recaudación en MM \$ por tipo de Impuestos Ecuador 2012-2017

\begin{tabular}{|c|c|c|c|c|c|c|c|c|}
\hline \multicolumn{7}{|c|}{ Relación recaudación en Millones de \$ por tipo de Impuestos 2012 - 2017} \\
\hline $\begin{array}{c}\text { Año/Tipo } \\
\text { Impuesto }\end{array}$ & ISLR & $\%$ & IVA & $\%$ & $\begin{array}{c}\text { Consumos } \\
\text { Especiales }\end{array}$ & $\%$ & $\begin{array}{c}\text { Salida de } \\
\text { Divisas }\end{array}$ & $\%$ \\
\hline $\mathbf{2 0 1 2}$ & 3.391 .237 & & 5.498 .240 & & 684.503 & & 1.159 .590 & \\
\hline $\mathbf{2 0 1 3}$ & 3.933 .236 & $\mathbf{1 5 , 9 8}$ & 6.186 .299 & $\mathbf{1 2 , 5 1}$ & $\mathbf{7 4 3 . 6 2 6}$ & $\mathbf{8 , 6 4}$ & 1.224 .592 & $\mathbf{5 , 6 1}$ \\
\hline $\mathbf{2 0 1 4}$ & 4.273 .914 & $\mathbf{8 , 6 6}$ & 4.512 .571 & $\mathbf{- 2 7 , 0 6}$ & 615.357 & $\mathbf{- 1 7 , 2 5}$ & 1.259 .690 & $\mathbf{2 , 8 7}$ \\
\hline $\mathbf{2 0 1 5}$ & 4.883 .112 & $\mathbf{1 4 , 2 5}$ & 4.778 .258 & $\mathbf{5 , 8 9}$ & 697.669 & $\mathbf{1 3 , 3 8}$ & 1.093 .977 & $\mathbf{- 1 3 , 1 6}$ \\
\hline $\mathbf{2 0 1 6}$ & 3.946 .284 & $\mathbf{- 1 9 , 1 9}$ & 5.704 .147 & $\mathbf{1 9 , 3 8}$ & 798.330 & $\mathbf{1 4 , 4 3}$ & 964.659 & $\mathbf{- 1 1 , 8 2}$ \\
\hline $\mathbf{2 0 1 7}$ & 4.177 .295 & $\mathbf{5 , 8 5}$ & 6.314 .931 & $\mathbf{1 0 , 7 1}$ & 949.402 & $\mathbf{1 8 , 9 2}$ & 1.097 .642 & $\mathbf{1 3 , 7 9}$ \\
\hline
\end{tabular}

Fuente: (SRI, 2012 - 2017). Elaboración Propia.

Como se puede apreciar con datos aportados por el Sistema de Rentas de Internas de Ecuador (SRI), se presenta la recaudación por tipo de impuestos en los periodos del 2012 -2017, ha habido importantes avances en cuanto a las metas propuestas y la recaudación como tal, en lo que tiene que ver la Impuesto de Rentas Recaudados hay un crecimiento sostenido entre el año 2012 al 2015 de un 44,99\%, habiendo una disminución del 19,19\% en el año 2016 (sin embargo esta reducción es mayor en \$ que la recaudación del año 2012), repuntando en un 5,85\% en el año 2017. En lo que tiene que ver al Impuesto al Valor Agregado (IVA), aun habiendo un decrecimiento en el año 2014 después de dos aumentos consecutivos (2012 y 2013), en general hubo un aumento en recaudación sostenido para el periodo en estudio de un $14,85 \%$. En lo que tiene que ver en el Impuesto a Consumos Especiales (Bebidas, cigarrillos, Armas, Perfumes, 


\section{La generación y administración de los recursos públicos en Ecuador, una perspectiva general}

Vol. 2, núm. 1., (2018)

Pablo Vicente Tejada Hinojosa; Alex Leopoldo Luque Letechi; Guillermo Roberto Abad Alvarado

entre otros) se observa el mismo comportamiento, solo un decrecimiento para el año 2014, pero a nivel de recaudación general desde el 2012 hasta el año 2017 presenta un porcentaje de aumento del 38,69\%, explicado por la reducción del consumo de algunos rubros, que integran a nivel general este impuesto. En lo que tiene que ver al Impuesto por Salida de Divisas. En líneas generales presenta el mismo comportamiento un aumento sostenido hasta el año 2014, disminución en los años 2015 y 2016 para tener un pequeño repunte en el año 2017 del 13,79\%.

Este es un impuesto que se cobra por las transacciones que se generan desde el Ecuador al exterior.

\begin{tabular}{|c|c|c|c|c|c|c|}
\hline Provincia & 2012 & 2013 & 2014 & 2015 & 2016 & 2017 \\
\hline Azuay & \begin{tabular}{|c|}
457.116 .59 \\
5,94
\end{tabular} & $\begin{array}{c}405.877 .70 \\
4,89\end{array}$ & \begin{tabular}{|c|}
435.819 .38 \\
9,05
\end{tabular} & $\begin{array}{c}499.284 \\
369\end{array}$ & $\begin{array}{c}487.292 . \\
150\end{array}$ & $\begin{array}{c}473.896 . \\
303\end{array}$ \\
\hline$\%$ & & $-11,21$ & 7,38 & 14,56 & $-2,40$ & $-2,75$ \\
\hline Bolívar & \begin{tabular}{|c|}
$9.783 .730,5$ \\
5 \\
\end{tabular} & \begin{tabular}{|c|}
10.896 .135 \\
68 \\
\end{tabular} & \begin{tabular}{|c|} 
12.339.427 \\
07
\end{tabular} & $\begin{array}{c}10.675 .9 \\
08 \\
\end{array}$ & $\begin{array}{c}11.837 .9 \\
18 \\
\end{array}$ & $\begin{array}{c}11.301 .0 \\
69\end{array}$ \\
\hline$\%$ & & 11,37 & 13,25 & $-13,48$ & 10,88 & $-4,53$ \\
\hline Cañar & \begin{tabular}{|c|}
$\begin{array}{c}30.865 .781 \\
63\end{array}$ \\
\end{tabular} & \begin{tabular}{|c|}
39.708 .156 \\
83 \\
\end{tabular} & \begin{tabular}{|c|}
37.048 .930 \\
37 \\
\end{tabular} & $\begin{array}{c}34.098 .7 \\
38 \\
\end{array}$ & $\begin{array}{c}32.575 .6 \\
70 \\
\end{array}$ & $\begin{array}{c}33.147 .6 \\
67 \\
\end{array}$ \\
\hline$\%$ & & 28,65 & $-6,70$ & $-7,96$ & $-4,47$ & 1,76 \\
\hline Carchi & $\begin{array}{c}16.725 .023 \\
82 \\
\end{array}$ & $\begin{array}{c}\text { 19.053.173, } \\
45 \\
\end{array}$ & \begin{tabular}{|c|}
21.491 .747 \\
36 \\
\end{tabular} & \begin{tabular}{|c|}
20.664 .4 \\
23 \\
\end{tabular} & $\begin{array}{c}18.865 .3 \\
22 \\
\end{array}$ & $\begin{array}{c}18.889 .8 \\
53 \\
\end{array}$ \\
\hline$\%$ & & 13,92 & 12,80 & $-3,85$ & $-8,71$ & 0,13 \\
\hline Chimborazo & $\begin{array}{c}\begin{array}{c}48.316 .086 \\
21\end{array} \\
\end{array}$ & \begin{tabular}{|c|}
47.797 .961$, \\
26 \\
\end{tabular} & $\begin{array}{c}59.096 .829 \\
50 \\
\end{array}$ & $\begin{array}{c}63.659 .9 \\
39 \\
\end{array}$ & $\begin{array}{c}65.462 .3 \\
96 \\
\end{array}$ & $\begin{array}{c}61.734 .8 \\
75 \\
\end{array}$ \\
\hline$\%$ & & $-1,07$ & 23,64 & 7,72 & 2,83 & $-5,69$ \\
\hline Cotopaxi & \begin{tabular}{|c|}
$\begin{array}{c}\text { 44.682.435, } \\
02\end{array}$ \\
\end{tabular} & $\begin{array}{c}52.861 .349, \\
09 \\
\end{array}$ & \begin{tabular}{|c|} 
54.494.111, \\
77 \\
\end{tabular} & $\begin{array}{c}68.388 .6 \\
44 \\
\end{array}$ & $\begin{array}{c}82.472 .6 \\
81 \\
\end{array}$ & $\begin{array}{c}86.708 .1 \\
24 \\
\end{array}$ \\
\hline$\%$ & & 18,30 & 3,09 & 25,50 & 20,59 & 5,14 \\
\hline El Oro & \begin{tabular}{|c|}
118.709 .89 \\
3,87 \\
\end{tabular} & \begin{tabular}{|c|}
132.675 .52 \\
2,04 \\
\end{tabular} & \begin{tabular}{|c|}
166.360 .50 \\
6,16 \\
\end{tabular} & $\begin{array}{c}185.354 \\
329 \\
\end{array}$ & $\begin{array}{c}183.716 . \\
074\end{array}$ & $\begin{array}{c}176.451 . \\
135\end{array}$ \\
\hline$\% \%$ & & 11,76 & 25,39 & 11,42 & $-0,88$ & $-3,95$ \\
\hline \multirow[t]{2}{*}{ Esmeraldas } & \begin{tabular}{|c|}
39.157 .518 \\
35 \\
\end{tabular} & $\begin{array}{c}44.056 .696, \\
03\end{array}$ & \begin{tabular}{|c|}
50.514 .146 \\
21 \\
\end{tabular} & $\begin{array}{c}56.255 .9 \\
16 \\
\end{array}$ & $\begin{array}{c}69.991 .3 \\
60 \\
\end{array}$ & $\begin{array}{c}54.380 .0 \\
23 \\
\end{array}$ \\
\hline & & 12,51 & 14,66 & 11,37 & 24,42 & $-22,30$ \\
\hline Galápagos & $\begin{array}{c}12.564 .330 \\
66\end{array}$ & $\begin{array}{c}14.000 .312 \\
27\end{array}$ & \begin{tabular}{|c|}
22.173 .824 \\
51
\end{tabular} & $\begin{array}{c}27.222 .3 \\
23\end{array}$ & $\begin{array}{c}30.007 .8 \\
37\end{array}$ & $\begin{array}{c}27.306 .7 \\
07\end{array}$ \\
\hline
\end{tabular}




\section{La generación y administración de los recursos públicos en Ecuador, una}

perspectiva general

Vol. 2, núm. 1., (2018)

Pablo Vicente Tejada Hinojosa; Alex Leopoldo Luque Letechi; Guillermo Roberto Abad

Alvarado

\begin{tabular}{|c|c|c|c|c|c|c|}
\hline$\%$ & & 11,43 & 58,38 & 22,77 & 10,23 & $-9,00$ \\
\hline Guayas & $\begin{array}{c}.280 .185 .1 \\
44,80\end{array}$ & $\begin{array}{c}\mathbf{3 . 1 5 7 . 7 1 7 . 8} \\
89,78 \\
\end{array}$ & \begin{tabular}{|c|}
3.204 .288 .0 \\
31,09
\end{tabular} & $\begin{array}{c}3.533 .06 \\
1.825 \\
\end{array}$ & $\begin{array}{c}3.429 .54 \\
7.691 \\
\end{array}$ & $\begin{array}{c}3.423 .63 \\
6.507 \\
\end{array}$ \\
\hline$\%$ & & $-3,73$ & 1,47 & 10,26 & $-2,93$ & $-0,17$ \\
\hline Imbabura & $\begin{array}{c}55.432 .125 \\
49 \\
\end{array}$ & \begin{tabular}{|c|}
84.741 .352 \\
24 \\
\end{tabular} & \begin{tabular}{|c|} 
99.663.062, \\
57 \\
\end{tabular} & $\begin{array}{c}110.949 \\
907 \\
\end{array}$ & $\begin{array}{c}100.466 \\
430 \\
\end{array}$ & $\begin{array}{c}92.376 .7 \\
24 \\
\end{array}$ \\
\hline$\%$ & & $\mathbf{5 2 , 8 7}$ & 17,61 & 11,33 & $-9,45$ & $-8,05$ \\
\hline Loja & $\begin{array}{c}\text { 61.426.402, } \\
31 \\
\end{array}$ & \begin{tabular}{|c|}
64.753 .586 \\
62 \\
\end{tabular} & $\begin{array}{c}\text { 67.760.677, } \\
99 \\
\end{array}$ & $\begin{array}{c}72.165 .1 \\
74 \\
\end{array}$ & $\begin{array}{c}74.363 .7 \\
07 \\
\end{array}$ & $\begin{array}{c}74.241 .4 \\
84 \\
\end{array}$ \\
\hline$\%$ & & 5,42 & 4,64 & $\mathbf{6 , 5 0}$ & 3,05 & $-0,16$ \\
\hline \multirow[t]{2}{*}{ Los Rios } & $\begin{array}{c}\text { 49.914.798, } \\
64 \\
\end{array}$ & \begin{tabular}{|c|}
56.446 .765 \\
14 \\
\end{tabular} & \begin{tabular}{|c|} 
62.392.920, \\
77 \\
\end{tabular} & $\begin{array}{c}64.823 .2 \\
73 \\
\end{array}$ & $\begin{array}{c}70.288 .2 \\
95 \\
\end{array}$ & $\begin{array}{c}69.214 .2 \\
41 \\
\end{array}$ \\
\hline & & 13,09 & 10,53 & 3,90 & 8,43 & $-1,53$ \\
\hline Manabi & $\begin{array}{c}181.503 .18 \\
6,29\end{array}$ & \begin{tabular}{|c|}
198.533 .50 \\
4,44
\end{tabular} & $\begin{array}{c}214.487 .69 \\
9,64\end{array}$ & $\begin{array}{c}205.805 \\
305 \\
\end{array}$ & $\begin{array}{c}187.522 \\
862 \\
\end{array}$ & $\begin{array}{c}219.098 . \\
617 \\
\end{array}$ \\
\hline$\% \%$ & & $\mathbf{9 , 3 8}$ & 8,04 & $-4,05$ & $-8,88$ & 16,84 \\
\hline Morona Santiago & $\begin{array}{c}12.663 .646, \\
17 \\
\end{array}$ & \begin{tabular}{|c|}
14.416 .206 \\
20 \\
\end{tabular} & \begin{tabular}{|c|}
$\begin{array}{c}\text { 13.684.442, } \\
63\end{array}$ \\
\end{tabular} & $\begin{array}{c}13.357 .4 \\
22 \\
\end{array}$ & $\begin{array}{c}14.690 .3 \\
89 \\
\end{array}$ & $\begin{array}{c}15.015 .6 \\
25 \\
\end{array}$ \\
\hline$\%$ & & 13,84 & $-5,08$ & $-2,39$ & & \\
\hline Napo & $\begin{array}{c}10.235 .955 \\
33 \\
\end{array}$ & \begin{tabular}{|c|}
11.478 .013 \\
70 \\
\end{tabular} & $\begin{array}{c}12.799 .092 \\
96 \\
\end{array}$ & $\begin{array}{c}13.170 .8 \\
16\end{array}$ & $\begin{array}{c}12.092 .6 \\
87 \\
\end{array}$ & $\begin{array}{c}10.893 .9 \\
47 \\
\end{array}$ \\
\hline$\%$ & & 12,13 & 11,51 & 2,90 & $-8,19$ & $-9,91$ \\
\hline Orellana & $\begin{array}{c}\text { 19.581.559, } \\
42 \\
\end{array}$ & \begin{tabular}{|c|}
24.415 .899 \\
49 \\
\end{tabular} & \begin{tabular}{|c|}
31.244 .818 \\
62 \\
\end{tabular} & $\begin{array}{c}27.354 .0 \\
37 \\
\end{array}$ & $\begin{array}{c}24.236 .3 \\
98 \\
\end{array}$ & $\begin{array}{c}21.879 .5 \\
39 \\
\end{array}$ \\
\hline$\%$ & & 24,69 & 27,97 & $-12,45$ & $-11,40$ & $-9,72$ \\
\hline Pastaza & $\begin{array}{c}\text { 11.125.931, } \\
03 \\
\end{array}$ & \begin{tabular}{|c|}
$\begin{array}{c}12.384 .448 \\
64\end{array}$ \\
\end{tabular} & \begin{tabular}{|c|}
11.746 .242 \\
33 \\
\end{tabular} & $\begin{array}{c}11.548 .5 \\
40 \\
\end{array}$ & $\begin{array}{c}12.745 .9 \\
17 \\
\end{array}$ & $\begin{array}{c}11.463 .9 \\
01\end{array}$ \\
\hline$\%$ & & 11 & $-5,15$ & $-1,68$ & 10,37 & $-10,06$ \\
\hline Pichincha & $\begin{array}{c}5.959 .686 .4 \\
51,15 \\
\end{array}$ & \begin{tabular}{|c|}
5.778 .265 .8 \\
89,36 \\
\end{tabular} & \begin{tabular}{|c|}
6.130 .658 .8 \\
49,03 \\
\end{tabular} & \begin{tabular}{|c|}
6.333 .68 \\
8.011 \\
\end{tabular} & $\begin{array}{c}5.784 .00 \\
5.925 \\
\end{array}$ & $\begin{array}{c}\mathbf{5 . 7 4 7 . 8 0} \\
3.570 \\
\end{array}$ \\
\hline$\%$ & & & & & & $-0,63$ \\
\hline Santa Elena & $\begin{array}{c}\text { 19.255.763, } \\
56 \\
\end{array}$ & \begin{tabular}{|c|}
$\begin{array}{c}24.206 .309 \\
05\end{array}$ \\
\end{tabular} & \begin{tabular}{|c|}
24.929 .111 \\
24 \\
\end{tabular} & $\begin{array}{c}24.674 .9 \\
49 \\
\end{array}$ & $\begin{array}{c}24.592 .9 \\
11 \\
\end{array}$ & $\begin{array}{c}25.445 .9 \\
74 \\
\end{array}$ \\
\hline$\%$ & & 25,71 & 2,99 & $-1,02$ & $-0,33$ & 3,47 \\
\hline \multirow[t]{2}{*}{$\begin{array}{l}\text { Santo Domingo De } \\
\text { Los Tsachilas }\end{array}$} & $\begin{array}{c}\text { 50.390.706, } \\
11 \\
\end{array}$ & \begin{tabular}{|c|}
54.361 .153 \\
60
\end{tabular} & $\begin{array}{c}\text { 57.043.629, } \\
63\end{array}$ & $\begin{array}{c}66.692 .2 \\
55 \\
\end{array}$ & $\begin{array}{c}68.967 .5 \\
41 \\
\end{array}$ & $\begin{array}{c}68.001 .3 \\
32 \\
\end{array}$ \\
\hline & & $\mathbf{7 , 8 8}$ & 4,93 & 16,91 & 341 & $-1,40$ \\
\hline Sucumbíos & $\begin{array}{c}21.033 .303, \\
76 \\
\end{array}$ & \begin{tabular}{|c|}
24.046 .311$, \\
38 \\
\end{tabular} & \begin{tabular}{|c|}
29.276 .593 \\
46
\end{tabular} & $\begin{array}{c}24.743 .1 \\
86 \\
\end{array}$ & $\begin{array}{c}22.716 .8 \\
76 \\
\end{array}$ & $\begin{array}{c}18.149 .7 \\
00\end{array}$ \\
\hline$\%$ & & 14,32 & & $-15,48$ & & $-20,10$ \\
\hline Tungur: & $\begin{array}{c}147.076 .36 \\
1,81 \\
\end{array}$ & \begin{tabular}{|c|}
136.123 .69 \\
6,41
\end{tabular} & \begin{tabular}{|c|}
144.683 .04 \\
1,81 \\
\end{tabular} & $\begin{array}{c}165.386 . \\
085\end{array}$ & $\begin{array}{c}170.416 \\
673 \\
\end{array}$ & $\begin{array}{c}161.691 . \\
737\end{array}$ \\
\hline$\%$ & & $-7,45$ & 6,29 & 14,31 & 3,04 & $-5,12$ \\
\hline Zamora Chinchipe & $\begin{array}{c}66.383 .815, \\
35 \\
\end{array}$ & \begin{tabular}{|c|}
$\begin{array}{c}19.319 .040 \\
48\end{array}$ \\
\end{tabular} & $\begin{array}{c}47.801 .793 \\
65\end{array}$ & $\begin{array}{c}30.931 .4 \\
28 \\
\end{array}$ & $\begin{array}{c}61.570 .9 \\
99 \\
\end{array}$ & $\begin{array}{c}61.206 .3 \\
21 \\
\end{array}$ \\
\hline$\%$ & & $-70,90$ & 147,43 & $-35,29$ & 99,06 & $-0,59$ \\
\hline No Definida & 65.106.916, & 65.264.371, & 69.166.694, & 79.429 .8 & 94.635 .9 & 74.350 .8 \\
\hline
\end{tabular}




\section{La generación y administración de los recursos públicos en Ecuador, una}

perspectiva general

Vol. 2, núm. 1., (2018)

Pablo Vicente Tejada Hinojosa; Alex Leopoldo Luque Letechi; Guillermo Roberto Abad Alvarado

\begin{tabular}{|l|l|l|l|l|l|l|l}
\hline & 59 & 66 & 78 & 78 & 21 & 39 \\
\hline
\end{tabular}

\section{Tabla $N^{\circ}$ 2: Recaudación en MM\$ por Impuestos Provincias de Ecuador periodo 2012-2017}

Fuente: (SRI, 2012 - 2017). Elaboración Propia.

En la tabla $\mathbf{N}^{\circ} \mathbf{2}$ se puede observar los ingresos por parte de impuestos generados o recaudados por las provincias Ecuatorianas, en general hay muchas fluctuaciones en los ingresos por años, solo se destaca la provincia de Cotopaxi que ha tenido un crecimiento sostenido desde los últimos 5 años, de resto se observa como en las provincias de Cañar, Carchi, Manabí, Orellana, Santa Elena, Sucumbíos, hubo disminuciones consecutivas de ingresos en los años 2015 y 2016. En las provincias de Esmeraldas, Galápagos, Loja y Santo Domingo De Los Tsachilas se puede observar que hubo aumentos sostenidos desde el año 2012 hasta el año 2016, habiendo disminuciones en el año 2017, en el resto de las provincias siguen habiendo fluctuaciones lo que hace muy difícil hacer un análisis, solo se evidencia que las disminuciones pueden haber sido generadas por situaciones internas de cada provincia en lo que tiene que ver con los impuestos que le corresponden, otros casos pueden estar solo relacionados a situaciones nacionales.

\section{Resultados de otros autores}

- La recaudación de impuestos en Ecuador pasó de USD 15558 millones a USD 14017 millones en el periodo enero-diciembre de 2016 en comparación con el mismo lapso del 2015. Esto representó un 9\% menos, según cifras publicadas por el Servicio de Rentas Internas 


\section{La generación y administración de los recursos públicos en Ecuador, una \\ perspectiva general}

Vol. 2, núm. 1., (2018)

Pablo Vicente Tejada Hinojosa; Alex Leopoldo Luque Letechi; Guillermo Roberto Abad

Alvarado

(SRI), los tributos más importantes arrastraron las recaudaciones a la baja: el impuesto a la renta (IR), el impuesto al valor agregado (IVA) por operaciones internas y el impuesto a la salida de divisas (ISD). Estos registraron una caída que está entre el 8 y 17\%. Esto evidencia una reducción en el dinamismo y actividad económica del país (Fernanda, Letty, \& Marco, 2018).

\section{Conclusiones.}

- A modo de conclusión general, en las tablas de recaudación aquí mostradas no se observa la cantidad de contribuyentes que cancelan los tributos, solo la información está reflejada en dólares. De esa manera no se puede evaluar la eficacia de una política tributaria, ya que lo que se busca es la creación de una conciencia tributaria para que la mayor cantidad de personas cumplan con su deber tributario.

\section{Conclusiones de otros autores.}

- La Auditoría Interna permanente debe funcionar como una actividad concebida para agregar valor optimizando las operaciones de una organización, así como contribuir al cumplimiento de sus objetivos; aportando un enfoque sistemático para evaluar y mejorar la eficacia de los procesos de gestión de riesgos, control y dirección (Abad \& Paúl, 2012).

- En el campo tributario, el hecho de que la administración tributaria disponga la información necesaria, mediante un banco de datos para el desarrollo de su gestión, éste es de gran importancia y más aún constituye un elemento indispensable, lo cual le permitirá ejercitar en 


\section{La generación y administración de los recursos públicos en Ecuador, una perspectiva general}

Vol. 2, núm. 1., (2018)

Pablo Vicente Tejada Hinojosa; Alex Leopoldo Luque Letechi; Guillermo Roberto Abad Alvarado

debida forma, acciones de control directas e identificadas, no con un criterio general, aplicando en forma indiscriminada (Reyes, 2005).

- Los impuestos directos superan a los indirectos como una consecuencia de las reformas tributarias que el gobierno del Eco. Rafael Correa ha impulsado como medida para contrarrestar la evasión e incrementar los impuestos para financiar los diversos programas sociales que ha implementado (Tito \& Liliana, 2015).

- El Presupuesto permite visualizar cuáles son los objetivos que persiguen las políticas públicas en busca del desarrollo y bienestar social, y como se financian dichos objetivos. Está conformado por los ingresos y gastos fiscales, los ingresos pueden ser corrientes o de capital y no dan lugar a obligación de reembolso; mientas que los gastos pueden ser corrientes, de inversión o capital (Benalcázar, 2013).

\section{Recomendaciones}

- Como recomendación general, la base de una cultura tributaria es generar la mayor cantidad de ingresos por medio de una mayor cantidad de contribuyentes que paguen, es por ello que hay que generar conciencia en explicar la importancia que tienen los tributos, los beneficios de pagarlos y su uso.

- Debe haber contraloría social, los contribuyentes deben velar por el buen gasto de los impuestos que se pagan, hay que estar alertas de los planes y proyectos que los gobiernos locales, e incluso el gobierno nacional hayan presupuestado para su ejecución y a la hora de no haberse ejecutado ningún proyecto exigir explicación del porqué de su no ejecución. 


\section{La generación y administración de los recursos públicos en Ecuador, una \\ perspectiva general}

Vol. 2, núm. 1., (2018)

Pablo Vicente Tejada Hinojosa; Alex Leopoldo Luque Letechi; Guillermo Roberto Abad

Alvarado

- Así mismo, los entes de control fiscal y de auditorías tanto de los gobiernos locales y el gobierno nacional, deben de igual manera hacerle seguimiento a los dineros que entran en las cuentas nacionales y municipales y hacia que partidas se distribuyen, y de igual forma revisar los presupuestos anuales para comparar lo planificado versus ejecutado.

- Se debe hacer una capacitación continua y permanente de los encargados del manejo de los dineros públicos, a veces por omisión o falta de preparación se incurre en delitos tipificados en ley.

- Se debe seguir implementando la automatización de los procesos de pago por internet (pago electrónico), lo que disminuye la burocracia en las oficinas de la administración pública y permite tener un mayor control y hacerle seguimiento a los fondos recaudados por tributos.

- Las estadísticas nacionales y municipales deben incluir la cantidad de contribuyentes que cumplen con su deber tributario. Si la política tributaria está dirigida a que más personas paguen, la manera de evaluarlo es indicando las cifras para hacer los correctivos necesarios, de estar por debajo de las proyecciones de recaudación, de tenerlas.

\section{Recomendaciones de otros autores}

- Al Gobierno Nacional, el establecimiento de la lucha anticorrupción como política de Estado, procediendo primeramente a realizar una amplia tarea de diagnóstico de los puntos más críticos en materia de deficiencias en la administración pública, y creando todo un conjunto de proyectos destinados a controlar el manejo responsable, racional y eficiente de los recursos públicos (Abad \& Paúl, 2012). 


\section{La generación y administración de los recursos públicos en Ecuador, una perspectiva general}

Vol. 2, núm. 1., (2018)

Pablo Vicente Tejada Hinojosa; Alex Leopoldo Luque Letechi; Guillermo Roberto Abad Alvarado

- A todas las personas particulares que en calidad de directivos, miembros o empleados que manejen recursos públicos, poner el mayor cuidado y diligencia en dicho asunto, teniendo siempre presente que dichos recursos pertenecen a todo el pueblo ecuatoriano (Abad \& Paúl, 2012).

- En conclusión se podría afirmar que la mejor forma de ejercer un acertado y adecuado control de cumplimiento de las disposiciones tributarias, por parte de los contribuyentes, sería mediante la estructuración de un banco de datos, que contengan toda la información necesaria, la misma que permita realizar todos los cruces de información posibles, aplicables a cada tipo de tributo y a toda clase de contribuyentes, por lo tanto es prudente recomendar que la administración tributaria, debe aunar todo tipo de esfuerzos, con la finalidad de llegar a la meta propuesta contando con un banco universal de datos (Reyes, 2005).

- Por último, para combatir la evasión y aumentar la eficacia de la fiscalización, existe otra alternativa de simplificar las normas tributarias, su pago y su fiscalización. Parece ser que ha llegado el momento de establecer una estructura de impuestos simple en cuanto a que el Estado pueda fácilmente recaudar los impuestos y en cuanto a que los contribuyentes dediquen el mínimo de energía y recursos posibles al proceso de pago de impuestos. Impuestos que tiendan a ser más uniformes, esto es, de tasas más parejas para todos los contribuyentes y que graven bases imponibles amplias, pueden ser el camino para lograr un sistema tributario capaz de enfrentar los grandes desafíos del país actual (Reyes, 2005). 


\section{La generación y administración de los recursos públicos en Ecuador, una \\ perspectiva general}

Vol. 2, núm. 1., (2018)

Pablo Vicente Tejada Hinojosa; Alex Leopoldo Luque Letechi; Guillermo Roberto Abad

Alvarado

- Considerar al momento de formular reformas a las Leyes Tributarias, un análisis minucioso y también proactivo a las diferentes variables económicas, sociales, comerciales, laborales, entre otras, de modo que atiendan a necesidades no sólo a corto plazo, sino que también sean sostenibles en el tiempo procurando de esta forma mantener una estabilidad normativa, lo que repercutirá directamente en una reducción de costos indirectos incurridos por el contribuyente en el cumplimiento de sus obligaciones (Tito \& Liliana, 2015).

\section{Bibliografía.}

Abad, G., \& Paúl, E. (2012). Insuficiencia jurídica de las auditorías internas en las instituciones públicas como mecanismo de control. Loja: Universiadad Nacional de Loja.

Benalcázar, J. (2013). La autonomía financiera municipal en el Ecuador, con especial referencia a los ingresos tributarios ya la potestad tributaria de los municipios. Revista IUS, 7(32), $27-40$.

Blacio, R. (2010). El tributo en el Ecuador. Revista de la Facultad de Derecho de México, 13(75).

Fernanda, H., Letty, M., \& Marco, S. (2018). Evolución del sistema tributario ecuatoriano y su influencia en el presupuesto general del Estado. Revista Observatorio de la Economía Latinoamericana, 2(1), 2-12.

Oyervide, F. (2012). La capacidad fiscal de los municipios como gobiernos autónomos descentralizados en Ecuador.i Gestión tributaria o pereza fiscal? Revista de Ciencias de la Administración y Economía.

Reyes, P. (2005). Alternativas para controlar la evasión tributaria en el Ecuador.

SRI. (2012 - 2017). Estadísticas Generales de Recaudación.

Tito, P., \& Liliana, E. (2015). Evolución de los impuestos directos e indirectos dentro del régimen tributario ecuatoriano y su recaudación en el período 2007-2012. 